\title{
Optimal Post-Detection Integration Technique for the Reacquisition of Weak GNSS Signals
}

\author{
David Gómez-Casco, José A. López-Salcedo and Gonzalo Seco-Granados \\ IEEC-CERES, Universitat Autònoma de Barcelona (UAB), Spain \\ Email: david.gomez.casco@uab.cat,jose.salcedo@uab.cat,gonzalo.seco@uab.cat
}

\begin{abstract}
This paper tackles the problem of finding the optimal non-coherent detector for the reacquisition of weak Global Navigation Satellite System (GNSS) signals in the presence of bits and phase uncertainty. Two solutions are derived based on using two detection frameworks: the Bayesian approach and the generalized likelihood ratio test (GLRT). We also derive approximate detectors of reduced computation complexity and without noticeable performance degradation. Simulation results reveal a clear improvement of the detection probability for the proposed techniques with respect to the conventional detectors implemented in high sensitivity GNSS (HS-GNSS) receivers to acquire weak GNSS signals. Finally, we draw conclusions on which is the best technique to reacquire weak GNSS signals in practice considering a trade-off between performance and complexity.
\end{abstract}

Index Terms

Bayesian approach, GLRT, HS-GNSS receivers, post-detection integration techniques, ROC curves.

\section{INTRODUCTION}

Nowadays, Global Navigation Satellite System (GNSS) receivers are used in a continuously increasing variety of applications, involving for instance car and pedestrian navigation. These receivers allow the user to know the position in open-sky conditions, where the signals coming from the satellites can be easily detected. Due to the success achieved by GNSS receivers in these conditions, a great interest to extend their applications to more challenging environments, which include indoor building, urban canyon and forested areas, has emerged [1].

However, the existence of obstacles in these environments causes a high attenuation of the received signal making the acquisition and tracking of weak GNSS signals a challenge. In this situation, conventional GNSS receivers are not usually able to detect the signals. This fact has led to the development of high 
sensitivity GNSS (HS-GNSS) receivers. These receivers usually acquire weak signals by extending the coherent integration time duration, which provides an additional gain in signal detection. Nevertheless, this duration cannot be increased without boundaries mainly due to the presence of a residual frequency offset and data bits. In these circumstances, if reliable signal detection requires a longer integration time than what is possible in a coherent manner, the receiver has to apply non-linear combinations of the coherent integration outputs, which are referred to as post-detection integration (PDI) techniques or noncoherent detectors. PDI techniques overcome the limitations of the coherent integration time duration by using a non-linear function. Although these techniques are less effective in accumulating signal energy than the coherent integration, they can use a longer integration time allowing the receiver to acquire satellites with lower carrier-to-noise ratio [2], [3].

Several PDI techniques have been proposed in the literature to acquire weak GNSS signals. The best known technique is the non-coherent PDI (NPDI) [4], which is robust against the presence of frequency offset and data bits. Another well-known option corresponds to the differential PDI (DPDI) [5], which is only robust against the presence of frequency offset, but provides better detection performance than the NPDI technique. An additional technique is the generalized PDI truncated (GPDIT) [6], which combines the two previous techniques. The GPDIT technique exhibits a gain in signal detection with respect to the performances of both the NPDI and DPDI techniques individually, even though it requires a larger computational load and is also only robust against frequency offset. Another alternative is the nonquadratic NPDI (NQ-NPDI) technique, which is robust against data bits and residual frequency offset [7]. This technique offers better signal detection improvement than the NPDI when the received signal can be acquired using a small number of non-coherent combinations. Recently, a detection technique has been presented in [8], which is robust against the presence of data bits but not against frequency offset. This technique consists in the combination between two detector the NPDI and a new one referred to as squaring detector.

As a matter of fact, although PDI techniques are usually implemented for detecting weak signals at the acquisition stage, they have received less attention for the reacquisition. A reacquisition must be carried out when the receiver has just lost the signal from one satellite owing to, for instance, strong attenuation caused by an obstacle in the path between the transmitter and the receiver. If the receiver loses the signal, it has to re-detect the signal in order to obtain the position of the user. However, the problem of detecting weak GNSS signals in the reacquisition is less complex than in the first acquisition since in case of reacquisition an accurate estimation of the Doppler frequency is available [9] and hence the most problematic impairment to extend the coherent integration duration are the data bits.

Despite the fact that some strategies have been proposed to detect weak GNSS signals, which are 
mentioned above, the optimal PDI technique for the reacquisition remains still unknown. This occurs because PDI techniques are designed for the first acquisition of the receiver, which has to mitigate the uncertainty of the Doppler frequency. For this reason, the purpose of this paper is to derive the optimal PDI technique by applying the detection theory tools for the reacquisition of weak GNSS signals. More precisely, the Bayesian approach and the generalized likelihood ratio test (GLRT) are used to formulate the detection problem and two PDI techniques are obtained, which require a significant amount of computational resources. We also present lower-complexity approximations of these two PDI techniques. Finally, the performance of the techniques proposed herein is compared to the PDI techniques used in previous work in terms of receiver operating characteristics (ROC) curves, revealing a clear gain in favour of our techniques.

The paper is organized as follows. Section $\amalg$ defines the signal model, while Section $\amalg$ makes a review of the most relevant PDI techniques implemented in HS-GNSS receivers. In Section IV new PDI techniques are derived using the Bayesian approach and the GLRT. Section $\nabla$ illustrates the simulation results based on ROC curves. Finally, Section VI draws the conclusions.

\section{Signal Model}

The first task of any GNSS receivers is to detect the satellites in view. To do so, a local replica of the transmitted signal from a satellite with tentative values of code delay and Doppler frequency is correlated with the signal received from the different satellites [10]. The result of this process is known as a crossambiguity function (CAF), which is computed for a given value of coherent integration time $\left(T_{\text {coh }}\right)$. Assuming that there is absence of navigation data bits transition, the CAF of one particular satellite can be expressed as [11]

$$
y\left(\tilde{\tau}, \tilde{f}_{d}\right)=A d e^{j \phi} \operatorname{sinc}\left(\Delta f T_{c o h}\right) r(\Delta \tau)+\omega
$$

where $\tilde{\tau}$ and $\tilde{f}_{d}$ are the tentative values of code-delay and Doppler frequency, respectively, $A$ is the received amplitude with phase $\phi, d$ is the data bit value that can be 1 or $-1, \Delta \tau=\tau-\tilde{\tau}$ is the residual delay offset between the local replica and the received GNSS signal, $\Delta f=f_{d}-\tilde{f}_{d}$ is the residual frequency offset, $r(\Delta \tau)$ is the normalized correlation function of the GNSS signal, and $\omega$ is additive white Gaussian noise (AWGN) with zero-mean and variance $\sigma^{2}$. The $\operatorname{sinc}\left(\Delta f T_{c o h}\right)$ term captures the degradation owing to the frequency offset between the local replica and the received signal.

The acquisition of a satellite provides a coarse estimation of code delay and Doppler frequency, which are obtained from the value of $\tilde{\tau}$ and $\tilde{f}_{d}$ that maximize the CAF. The accuracy of theses estimations can be improved performing a finer search of Doppler frequency and code delay in the CAF. Then, the 
incoming signal is tracked by correlating it with a local replica, which contains accurate estimations of Doppler frequency and code delay. This process is usually carried out for a long period of time. However, the tracking of the signal can be lost due to, for example, the attenuation caused by an obstacle between the satellite and the receiver. In this situation, the HS-GNSS receiver tries to reacquire the received signal from the satellite. To do so, a local replica, which includes the estimations of code delay and Doppler frequency obtained in the tracking stage before losing the signal, is correlated again with the received signal for different time instants, which becomes [12]

$$
y_{k}=I_{k}+j Q_{k}=A d_{k} e^{j \phi}+w_{k},
$$

where $I_{k}=\Re\left(y_{k}\right), Q=\Im\left(y_{k}\right), w_{k}$ is the noise component, the index $k=1, \ldots, N_{n c}$ represents the time instant when the correlator output $y_{k}$ is computed, $d_{k}$ are the data bits assumed to be a random variable taking values of 1 and -1 with the same probability. The amplitude $A$ and the phase $\phi$ are constant with $k$, and $w_{k}$ is assumed independent for each $k$, but identically distributed. It is worth mentioning that the correlation output $y_{k}$ is usually computed for several close values of the code delay estimation since this estimation may have changed slightly due to the movement of the satellite and the receiver. Nonetheless, we omit this dependence since we can consider we are performing the analysis only for one of these values.

Combinations of several correlator outputs are needed to detect the weak GNSS signal. The best way to obtain a gain in terms of signal detection is increasing the $T_{\text {coh }}$ (i.e. coherently combining different correlator outputs), though its duration is limited by data bits. If the coherent integration is not enough to detect the signal in harsh conditions, we must resort to apply PDI techniques, which provide signal detection improvements since they can increase the integration time by using a non-linear function. In order to known whether the satellite is present or not, the output of a PDI technique denoted as $L_{x}$ is compared to a signal detection threshold. If the magnitude of $L_{x}$ surpasses the detection threshold the satellite is considered to be present, but if this magnitude does not surpass the detection threshold, the satellite is assumed to be absent. A block diagram representing the reacquisition process is shown in Fig. 1. The problem of obtaining the optimal PDI technique consists in finding a function $f\left(y_{1}, \ldots, y_{N_{n c}}\right)$ that allows the receiver to discriminate between the two hypotheses $H_{0}$ (the satellite is absent) and $H_{1}$ (the satellite is present) with the lower probability of false alarm and greater probability of detection:

- Under $H_{0}: y_{k}=w_{k}$ is a complex Gaussian noise with mean zero and variance $\sigma^{2}$.

- Under $H_{1}: y_{k}=A d_{k} e^{j \phi}+w_{k}$ is the signal plus complex Gaussian noise.

It is worth mentioning that if the phase of the signal was time-varying, the signal detection problem would be completely different, which leads to other types of solutions. Examples of signal detection 


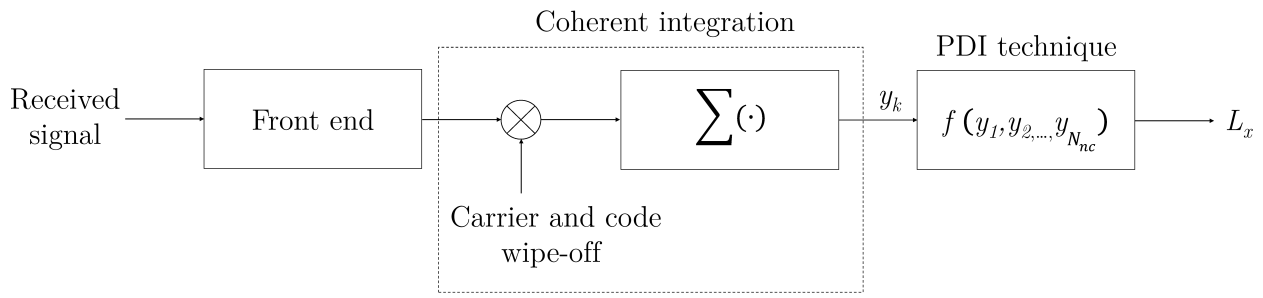

Fig. 1. Block diagram of the GNSS signal reacquisition.

problems with time-varying phase can be found in [7], [13].

\section{STATE-OF-THE-ART OF PDI TECHNIQUES FOR HS-GNSS RECEIVERS}

In this section we present a review of the most relevant PDI techniques implemented in HS-GNSS receivers, which will be used as a benchmark to compare the performance of the PDI techniques presented in Section IV. The optimal detector assuming a received signal that only contains an unknown phase during all the integration time is the coherent integration [14]

$$
L_{\mathrm{coh}}(\mathbf{y})=\left|\sum_{k=1}^{N_{n c}} y_{k}\right|,
$$

where $\mathbf{y} \doteq\left[y_{1}, \ldots, y_{N_{n c}}\right]^{T}$. However, the coherent integration is degraded, impaired in presence of frequency offset and data bits. In presence of these impairments, the most widely applied PDI technique is the NPDI, which is given by [4]

$$
L_{\mathrm{NPDI}}(\mathbf{y})=\sum_{k=1}^{N_{n c}}\left|y_{k}\right|^{2} .
$$

The NPDI technique is robust against the phase variations caused by data bits and frequency offset since it removes these variations by using the squared absolute value.

Alternatively, another technique to detect weak signals is the DPDI defined as follows [5]:

$$
L_{\text {DPDI }}(\mathbf{y})=\left|\sum_{k=2}^{N_{n c}} y_{k} y_{k-1}^{*}\right| .
$$

This technique usually offers better performance than the NPDI technique, but it experiences a performance degradation in presence of data bits. Another alternative is the NQ-NPDI technique [7]:

$$
L_{\mathrm{NQ}-\mathrm{NPDI}}(\mathbf{y})=\sum_{k=1}^{N_{n c}}\left|y_{k}\right| .
$$

The NQ-NPDI technique provides an improvement in signal detection performance over the NPDI technique, especially if the signal can be detected using a small number of $N_{n c}$, that is, $N_{n c} \leq 10$. 
Moreover, it is robust against frequency offset and data bits. An additional technique, denoted as GPDIT, combines the NPDI and DPDI techniques as [6]

$$
L_{\mathrm{GPDIT}}(\mathbf{y})=L_{\mathrm{NPDI}}(\mathbf{y})+2 L_{\mathrm{DPDI}}(\mathbf{y})=\sum_{k=1}^{N_{n c}}\left|y_{k}\right|^{2}+2\left|\sum_{k=2}^{N_{n c}} y_{k} y_{k-1}^{*}\right| .
$$

The GPDIT technique outperforms the NPDI and DPDI techniques as long as the signal does not contain data bits. This occurs because the GPDIT technique consists of the DPDI term, which suffers a significant degradation in presence of data bits.

\section{Detection STRATEgies}

This section uses two different detection strategies to find the optimal PDI technique for the signal model described in Section [II. These strategies are the Bayesian approach and the GLRT, which are usually applied in detection problems with unknown parameters.

\section{A. Bayesian approach}

The Bayesian approach is often used when likelihood ratio test (LRT) contains unknown parameters, to which a prior probability distribution can be assigned. Indeed, under these conditions, the Bayesian approach leads to the optimal detector [15]. This approach consists in calculating the expectation of the LRT with respect to the a priori distribution of the unknown parameter. More precisely, the difficulty caused by the unknown parameter is circumvented by averaging the conditional probability density function (PDF) to obtain the unconditional PDF, which does not depend on the unknown parameter. The conditional PDF of the correlators outputs assuming that these outputs include data bits uniformly distributed with equal probability is written under $H_{1}$ as [7]

$$
p\left(\mathbf{y} ; H_{1}, \phi\right)=\frac{1}{\left(\pi \sigma^{2}\right)^{N_{n c}}} \exp \left(-\sum_{k=1}^{N_{n c}} \frac{1}{\sigma^{2}}\left(I_{k}^{2}+Q_{k}^{2}+A^{2}\right)\right) \prod_{k=1}^{N_{n c}} \cosh \left(\frac{2 A}{\sigma^{2}}\left(I_{k} \cos (\phi)+Q_{k} \sin (\phi)\right)\right) .
$$

Under $H_{0}$, the PDF of $\mathbf{y}$ can be expressed as follows,

$$
p\left(\mathbf{y} ; H_{0}\right)=\frac{1}{\left(\pi \sigma^{2}\right)^{N_{n c}}} \exp \left(-\sum_{k=1}^{N_{n c}} \frac{1}{\sigma^{2}}\left(I_{k}^{2}+Q_{k}^{2}\right)\right) .
$$

The Bayesian approach is based on a ratio of the two PDFs above given by

$$
L_{B}(\mathbf{y})=\frac{\int p\left(\mathbf{y} ; H_{1}, \phi\right) p(\phi) d \phi}{p\left(\mathbf{y} ; H_{0}\right)}=\frac{p\left(\mathbf{y} ; H_{1}\right)}{p\left(\mathbf{y} ; H_{0}\right)} \lessgtr \tilde{\gamma}_{B},
$$

where $p(\phi)$ is the prior PDF of $\phi$ and $\tilde{\gamma}_{B}$ is the detection threshold. First, to apply the Bayesian approach we obtain an expression of the ratio between the two PDFs: $p\left(\mathbf{y} ; H_{1}, \phi\right)$ and $p\left(\mathbf{y} ; H_{0}\right)$. After removing some irrelevant constants, the ratio can be written as

$$
L_{B}^{\prime}(\mathbf{y}, \phi)=\prod_{k=1}^{N_{n c}} \cosh \left(\frac{2 A}{\sigma^{2}}\left(I_{k} \cos (\phi)+Q_{k} \sin (\phi)\right)\right)
$$


Second, we eliminate the phase information in (11) using the prior information. The prior PDF of $\phi$ is assumed to be a uniform random variable from $-\pi$ to $\pi$. The resulting Bayesian approach is given by the following expression:

$$
L_{B}^{\prime \prime}(\mathbf{y})=\frac{1}{2 \pi} \int_{-\pi}^{\pi} \prod_{k=1}^{N_{n c}} \cosh \left(\frac{2 A}{\sigma^{2}} c_{k}(\phi)\right) d \phi
$$

with

$$
c_{k}(\phi)=I_{k} \cos (\phi)+Q_{k} \sin (\phi) .
$$

Note that the larger the value of $N_{n c}$, the larger the number of multiplicative terms in the integral. To solve this integral, we apply the properties of the product of $\cosh$ functions, that is, $\cosh (x) \cosh (y)=$ $(\cosh (x+y)+\cosh (x-y)) / 2$. Proceeding in this way the integral can be rewritten as a series of integrals, where each one contains the cosh of a certain combination of sums and subtractions of the terms $c_{k}(\phi)$ as

$$
\begin{gathered}
L_{B}^{\prime \prime}(\mathbf{y})=\frac{1}{2 \pi 2^{N_{n c}-1}}\left(\int_{-\pi}^{\pi} \cosh \left(\frac{2 A}{\sigma^{2}}\left(c_{1}(\phi)+c_{2}(\phi)+\ldots+c_{N_{n c}}(\phi)\right)\right) d \phi+\ldots+\right. \\
\left.\int_{-\pi}^{\pi} \cosh \left(\frac{2 A}{\sigma^{2}}\left(c_{1}(\phi)-c_{2}(\phi)-\ldots-c_{N_{n c}}(\phi)\right)\right) d \phi\right),
\end{gathered}
$$

for which a more compact expression is

$$
L_{B}^{\prime \prime}(\mathbf{y})=\frac{1}{2 \pi 2^{N_{n c}-1}} \sum_{m=1}^{2^{N_{n c}-1}} \int_{-\pi}^{\pi} \cosh \left(\frac{2 A}{\sigma^{2}}\left(a_{m} \cos (\phi)+b_{m} \sin (\phi)\right)\right) d \phi,
$$

where $2^{N_{n c}-1}$ is the number of cosh functions that appear after applying the property of the multiplication of several cosh functions. The $a_{m}$ and $b_{m}$ coefficients aim at encompassing all possible combinations of additions and subtractions of $I_{k}$ and $Q_{k}$, respectively, excluding those that refer to others already considered but with opposite sign. By stacking the above-mentioned coefficients into vectors $\mathbf{a} \doteq\left[a_{1}, \cdots, a_{2 N_{n c}-1}\right]^{T}$ and $\mathbf{b} \doteq\left[b_{1}, \cdots, b_{2 N_{n c}-1}\right]^{T}$, we can compute their value as follows,

$$
\begin{aligned}
& \mathbf{a}=\mathbf{M I} \\
& \mathbf{b}=\mathbf{M Q},
\end{aligned}
$$

where $\mathbf{I} \doteq\left[I_{1}, \ldots, I_{N_{n c}}\right]^{T}, \mathbf{Q} \doteq\left[Q_{1}, \ldots, Q_{N_{n c}}\right]^{T}$, and $\mathbf{M}$ is a $\left(2^{N_{n c}-1} \times N_{n c}\right)$ matrix whose rows contain all the possible combinations of +1 and -1 , excluding those that differ from another row in a global change of sign as

$$
\mathbf{M} \doteq\left[\begin{array}{ccccc}
1 & -1 & -1 & \ldots & -1 \\
1 & 1 & -1 & \ldots & -1 \\
\vdots & \vdots & \vdots & \ddots & \vdots \\
1 & 1 & 1 & \ldots & 1
\end{array}\right]
$$


Now, the integral can be solved by the following procedure as

$$
\begin{aligned}
& L_{B}^{\prime \prime}(\mathbf{y})=\frac{1}{\pi 2^{N_{n c}}} \sum_{m=1}^{2^{N_{n c}-1}} \int_{-\pi}^{\pi} \cosh \left(\frac{2 A}{\sigma^{2}} \sqrt{a_{m}^{2}+b_{m}^{2}} \cos \left(\phi-\operatorname{atan}\left(\frac{b_{m}}{a_{m}}\right)\right)\right) d \phi \\
& =\frac{1}{\pi 2^{N_{n c}+1}} \sum_{m=1}^{2^{N_{n c}-1}}\left(\int_{-\pi}^{\pi} e^{\frac{2 A}{\sigma^{2}} \sqrt{a_{m}^{2}+b_{m}^{2}} \cos (\phi)} d \phi+\int_{-\pi}^{\pi} e^{-\frac{2 A}{\sigma^{2}} \sqrt{a_{m}^{2}+b_{m}^{2}} \cos (\phi)} d \phi\right) \\
& =\frac{1}{2^{N_{n c}-1}} \sum_{m=1}^{2^{N_{n c}-1}} I_{0}\left(\frac{2 A}{\sigma^{2}} \sqrt{a_{m}^{2}+b_{m}^{2}}\right),
\end{aligned}
$$

where $I_{0}$ denotes the zero-order modified Bessel function. Finally, removing some irrelevant constants the resulting detector can be expressed as

$$
L_{\mathrm{BAPDI}}(\mathbf{y})=\sum_{m=1}^{2^{N_{n c}-1}} I_{0}\left(\frac{2 A}{\sigma^{2}} \sqrt{a_{m}^{2}+b_{m}^{2}}\right) \lessgtr \gamma_{B},
$$

where $\gamma_{B}$ is the detection threshold. The result expressed in (20) is referred to as Bayesian approach PDI (BAPDI) technique. This technique is optimum in presence of unknowns bits and an unknown constant phase. Nonetheless, the BAPDI technique depends on the ratio of $A$ and $\sigma^{2}$. Despite the fact that some receivers can know this ratio in tracking stage since they use a carrier-to-noise estimator, the goal of this paper is to derive a detector which does not depends on the parameters $A$ and $\sigma^{2}$ so that it can be implemented in any receiver. To do so, we propose to apply the approximation of $I_{0}(x) \approx \exp (|x|)$, valid for relative large values of $x$. This approximation can be applied for our problem since the argument of (20) is not a small magnitude when the received signal has the same or similar combination of bits as one of the rows of the matrix $\mathbf{M}$. Then, by considering $I_{0}(x) \approx \exp (|x|)$, we get

$$
\sum_{m=1}^{2^{N_{n c}-1}} \exp \left(\left|\frac{2 A}{\sigma^{2}} \sqrt{a_{m}^{2}+b_{m}^{2}}\right|\right) \lessgtr \gamma_{B} .
$$

Introducing the logarithm of the LRT becomes

$$
\ln \left(\sum_{m=1}^{2^{N_{n c}-1}} \exp \left(\left|\frac{2 A}{\sigma^{2}} \sqrt{a_{m}^{2}+b_{m}^{2}}\right|\right)\right) \lessgtr \ln \left(\gamma_{B}\right) .
$$

To simplify the expression above, we make use of the log-sum-exp approximation, which consists in taking the maximum of the different exponentials. This approximation is reasonable for a high signalto-noise ratio (SNR) at the output of the PDI technique. Such values of SNR are usually obtained at this output because otherwise the signal could not be detected. In this situation, the largest term dominates in the sum of (22) as

$$
\max _{m}\left(\left|\frac{2 A}{\sigma^{2}} \sqrt{a_{m}^{2}+b_{m}^{2}}\right|\right) \lessgtr \gamma_{B}^{\prime} .
$$


The larger the deviation of the argument of (22), the better the approximation becomes. Finally, in-

corporating the now irrelevant constant $\frac{2|A|}{\sigma^{2}}$ into the threshold, the resulting detector can be expressed as

$$
L_{\mathrm{MBAPDI}}(\mathbf{y})=\max _{m}\left(\sqrt{a_{m}^{2}+b_{m}^{2}}\right) \lessgtr \gamma_{B}^{\prime \prime}
$$

The solution provided by (24) is referred to as maximum BAPDI (MBAPDI) technique. The MBAPDI technique can be implemented in any HS-GNSS receiver because it does not depend on the parameters $A$ and $\sigma^{2}$. It is worth mentioning that if chosen index $m$ corresponds to the correct sequence of bits, then the result would be the same as for the coherent detector in the hypothesis $H_{1}$, but this will not always happen due to the presence of noise. Moreover, although this happened, we would have a performance degradation with respect to the coherent detector. This is because the MBAPDI requires the use of the maximum function also in the hypothesis $H_{0}$, making the receiver choose the largest value among the different $2^{N_{n c}-1}$ samples of noise, which increases the number of false alarms.

\section{B. Generalized likelihood ratio test}

A common approach to design detectors with unknown parameters deals with the combination of estimation and detection. The best known joint estimation and detection approach is the GLRT, which consists of two steps. First, the maximum likelihood (ML) estimate of the unknown parameters are found. Second, the unknown parameters are replaced by their ML estimates under each hypothesis and the LRT is calculated as if the estimated parameters were correct [16], [17].

Although no claims about the optimality of the GLRT can be made, it provides good results in general. Moreover, the GLRT formulation usually provides simpler expressions than the Bayesian approach, which requires the integral of products of several PDFs. This occurs because ML estimation equations sometimes result in a closed-form solution. However, this is not the case of our problem where the ML estimate of the received phase affected by bits does not admit a closed-form solution. In this situation, two options are feasible: making an approximation of the ML equation in order to get a closed-form solution, which was done in [12] or using a one-dimensional search method to evaluate the ML estimate.

A PDI technique has been already obtained in [8] using an approximation of the ML phase estimate provided in [12] and replacing it in an expression of the LRT approximated for low SNR regime. Before proceeding, we make a brief description of the work done previously by others authors and after that we present new PDI techniques based on using different approaches of the GLRT. In [12], the authors 
computed the ML solution of the signal phase, which contains unknown bits, from the PDF of $\mathbf{y}$ as

$$
p\left(\mathbf{y} ; H_{1}, \phi\right)=\frac{1}{\left(\pi \sigma^{2}\right)^{N_{n c}}} \exp \left(-\sum_{k=1}^{N_{n c}} \frac{1}{\sigma^{2}}\left(I_{k}^{2}+Q_{k}^{2}+A^{2}\right)\right) \prod_{k=1}^{N_{n c}} \cosh \left(\frac{2 A}{\sigma^{2}}\left(I_{k} \cos (\phi)+Q_{k} \sin (\phi)\right)\right) .
$$

The log-likelihood function for $\phi$, removing the terms that are not affected by $\phi$, can be expressed as

$$
L(\mathbf{y}, \phi)=\sum_{k=1}^{N_{n c}} \ln \left(\cosh \left(\frac{2 A}{\sigma^{2}}\left(I_{k} \cos (\phi)+Q_{k} \sin (\phi)\right)\right)\right) .
$$

In order to find a closed-form solution of $\phi$ the $\ln (\cosh (x))$ function is approximated by $x^{2} / 2$. Thus, the closed-form expression of $\phi$ that approximately maximizes (26) is

$$
\hat{\phi}=\frac{1}{2} \operatorname{atan} 2\left(2 \sum_{k=1}^{N_{n c}} I_{k} Q_{k}, \sum_{k=1}^{N_{n c}} I_{k}^{2}-Q_{k}^{2}\right)
$$

where $\operatorname{atan} 2(x, y)$ is the four quadrant atan function.

Another way to find the value of $\phi$ that maximizes (26) is by using an iterative algorithm. It can be easily carried out implementing a one-dimensional search. The comparison between the estimators and the Cramer-Rao bound (CRB) is shown in Fig.2. The result illustrates that the ML estimate obtained by a one-dimensional search method exhibits practically the same performance as the approximation in (27). The CRB of the phase estimate is $1 /\left(2 \operatorname{SNR} N_{n c}\right)$ [18], where the SNR is defined as $A^{2} / \sigma^{2}$.

In [8], a PDI technique was presented based on the GLRT approach. The authors used the log-LRT, which can be expressed as (26). They propose to approximate $L(\mathbf{y}, \phi)$ defined in (26) for a low SNR regime applying a Taylor series of the $\ln (\cosh (x))$ function as $x^{2} / 2$, which leads to

$$
\sum_{k=1}^{N_{n c}}\left(\frac{2 A}{\sigma^{2}}\left(I_{k} \cos (\phi)+Q_{k} \sin (\phi)\right)\right)^{2} \lessgtr \gamma_{G}^{\prime} .
$$

Replacing the approximation of the phase estimate in (27) into (28), and making some simplifications, the NPDISD detector can be obtained as

$$
L_{\mathrm{NPDISD}}(\mathbf{y})=\sum_{k=1}^{N_{n c}}\left|y_{k}\right|^{2}+\left|\sum_{k=1}^{N_{n c}} y_{k}^{2}\right|
$$

The NPDISD detector consists of two non-coherent detectors or PDI techniques. The first detector is the conventional NPDI detector. The second detector is the squaring detector (SD), which consists in summing the squared complex correlator outputs. Despite the fact that this solution provides a good performance, an enhancement of this approach can be carried out since HS-GNSS receivers do not usually work in a very low SNR regime at the output of the coherent correlation. This occurs because the correlator outputs in HS-GNSS receivers are obtained using a long $T_{c o h}$ in general and combining few correlation outputs 


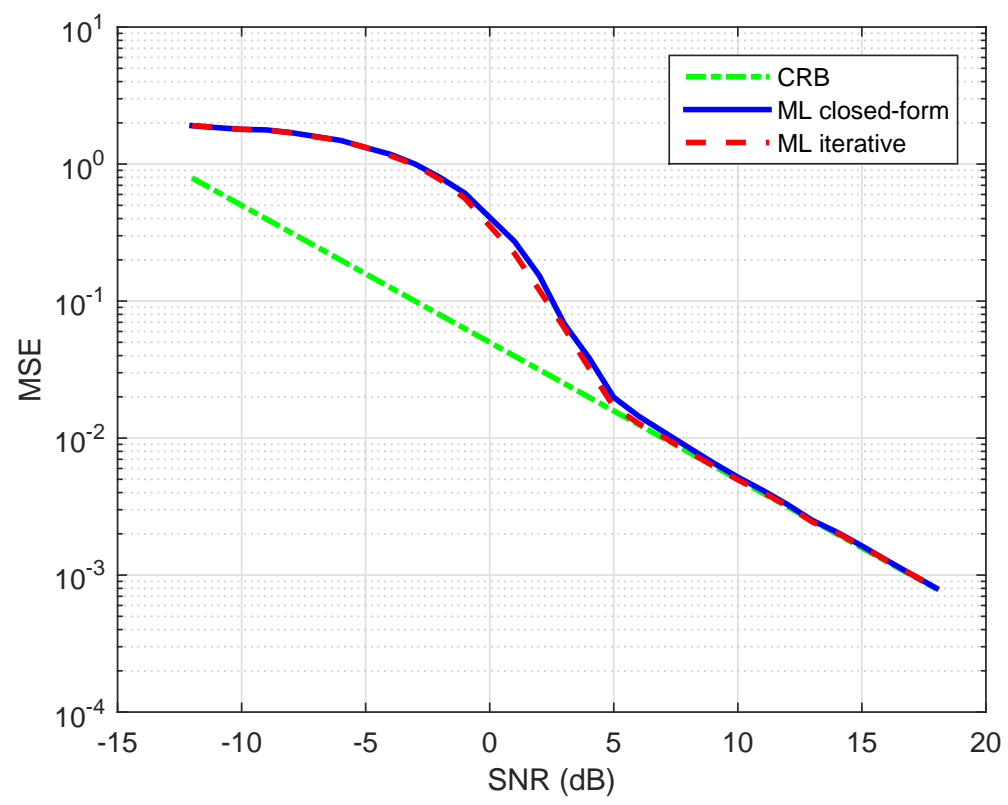

Fig. 2. Performance comparison of the different estimators and the CRB for $N_{n c}=10$. The approximation of the ML solution expressed in 27) is referred to as ML closed-form and the one obtained from a one-dimensional search is indicate as ML iterative.

the signal can be detected. Then, the approximation of Taylor series used in (28) for low SNR values might not be the best option to obtain the best performance of the GLRT approach.

For this reason, the purpose of the following subsections is to propose several new alternatives to the GLRT in order to enhance the performance of the NPDISD technique. More precisely, we present three new approaches to obtain the best detectors using the GLRT approach in the context of HS-GNSS receivers.

1) GLRT (strict): The first one boils down to the strict application of the GLRT approach. This approach is based on using the log-LRT and replacing the unknown parameter $\phi$ value by its ML estimation, which must be obtained from a one-dimensional search in (26), as

$$
L\left(\mathbf{y}, \hat{\phi}_{\mathrm{ML}}\right)=\sum_{k=1}^{N_{n c}} \ln \left(\cosh \left(\frac{2 A}{\sigma^{2}}\left(I_{k} \cos \left(\hat{\phi}_{\mathrm{ML}}\right)+Q_{k} \sin \left(\hat{\phi}_{\mathrm{ML}}\right)\right)\right)\right),
$$

where $\hat{\phi}_{\mathrm{ML}}$ is the ML estimate of $\phi$. This approach allow us to know, which is the optimal performance of the GLRT method and how far it is from the Bayesian approach. This is an important point because the outcome of the Bayesian approach is the optimal detector under the assumed conditions. As we have seen in Subsection IV-A the result of the Bayesian approach implies the computation of a matrix, whose 
size increases exponentially with the $N_{n c}$ value. In fact, the computation of this matrix can become a handicap. For this reason, if the difference between the performance of the Bayesian approach and the GLRT was quite similar, the application of the GLRT could be the best option. We will continue this discussion later on in the Section $\nabla$ where the performance comparison of the PDI techniques is analysed.

2) GLRT approximation in closed-form: The second approach is based on the log-LRT in (30), but reducing the complexity of this method to estimate $\phi$. Given the phase estimate in (27) exhibits almost the same performance as at ML phase estimate, while avoiding the one dimensional search, we propose to replace $\hat{\phi}_{\mathrm{ML}}$ in (30) with (27), resulting in:

$$
L(\mathbf{y}, \hat{\phi})=\sum_{k=1}^{N_{n c}} \ln \left(\cosh \left(\frac{2 A}{\sigma^{2}}\left(I_{k} \cos (\hat{\phi})+Q_{k} \sin (\hat{\phi})\right)\right)\right) .
$$

3) GLRT approximation for high SNR regime: The alternatives described in Subsection IV-B2 and Subsection IV-B1 require the knowledge of the SNR, $A / \sigma^{2}$. This is a drawback since this information is sometimes unknown by the receiver. For this reason, the last method that we propose avoids the need of knowing the SNR a priori. The way to obtain a detector that does not depend on the SNR is to adopt an approximation of the $\ln (\cosh (x))$ function as $|x|-\ln (2)$. This approximation gives an excellent fit for relative large values of $x$, which is a region appropriate to detect signals in the context of HS-GNSS receivers. After using this approximation, the PDI technique is independent of the scale factors $A$ and $\sigma^{2}$. Thus, the resulting technique can be expressed as

$$
L_{\mathrm{GLRT}_{a . l .}}(\mathbf{y}, \hat{\phi})=\sum_{k=1}^{N_{n c}}\left|I_{k} \cos (\hat{\phi})+Q_{k} \sin (\hat{\phi})\right| .
$$

This PDI technique may offer similar performance as the two previous techniques presented in Subsection IV-B1 and SubsectionIV-B2 when the SNR at the correlator output is relatively high. Besides not requiring the knowledge of the SNR, this technique avoids the use of two non-linear functions such as the ln and cosh. It is worth mentioning that (32) has some resemblance to the NQ-NPDI technique described in (6), which was derived for time-varying phase signals. The NQ-NPDI technique offers a great performance in scenarios where the SNR is relatively high and the received signal can suffer phase changes [7]. However, the technique proposed in this subsection is derived for signals with constant phase. This fact suggests that in scenarios where the received signal includes a constant phase, the detector in (32) could provide a promising performance.

\section{Simulation Results}

This section presents the simulation results based on receiver operating characteristic (ROC) curves. These curves compare the detection performance of the PDI techniques proposed herein to the more 


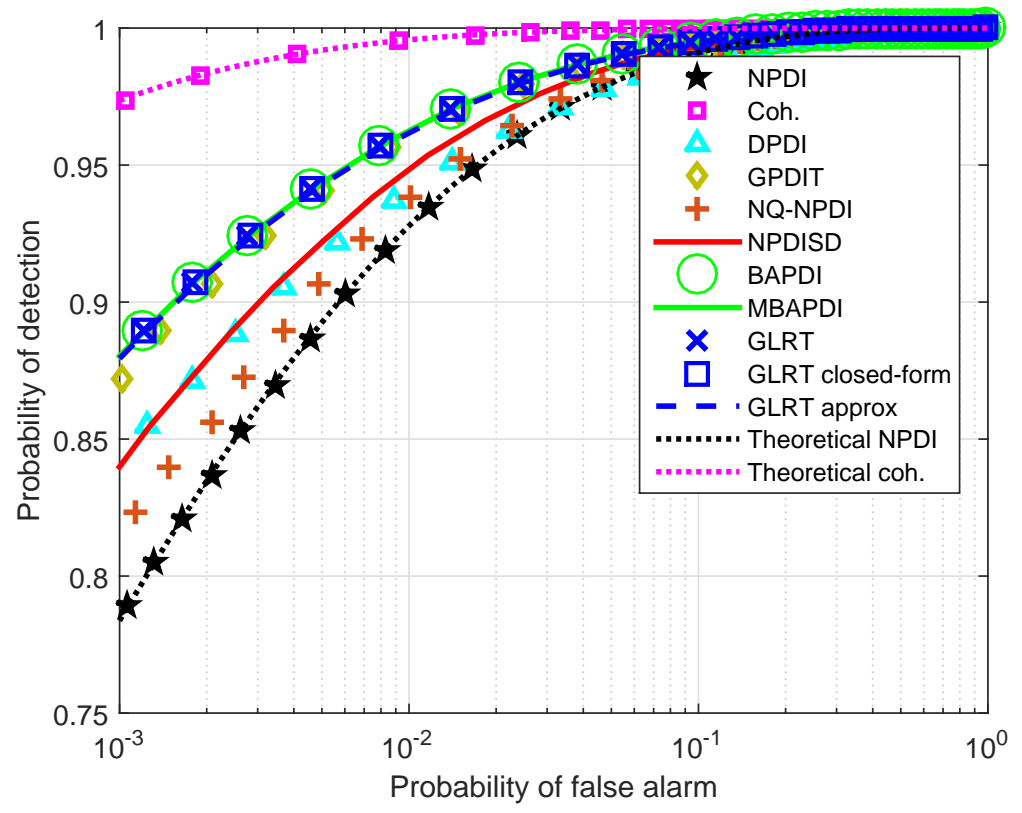

Fig. 3. Performance comparison of the different detectors in absence of bits for $N_{n c}=6, A=1.6$ and $\sigma=1$. In the legend, GLRT, GLRT closed-form and GLRT approx refer to the techniques explained in Subsections IV-B1, IV-B2 and IV-B3. respectively.

relevant PDI techniques found in the literature. Results are obtained using Monte Carlo simulations and the $\sigma$ value is normalized to 1 .

Fig. 3 shows the comparison among the different PDI techniques in an ideal channel containing only Gaussian noise and an unknown constant phase, but in absence of data bits in the received signal for $N_{n c}=6$ and $A=1.6$. As we expected, in this situation, the optimal detector is the coherent integration since there are not effects that pose limits on its duration. The worst performing technique corresponds to the NPDI technique. The proposed five techniques, namely, BAPDI, MBAPDI, and the three obtained from the GLRT method explained in Subsections IV-B1, IV-B2 and IV-B3, exhibit similar performance, which is also better than that of the DPDI, GPDIT, NPDISD and NQ-NPDI techniques. Theoretical ROC curves of the coherent integration and the NPDI technique are included to the figures, which are given by [14] and [19], respectively. However, for the rest of the detectors there are no known closed-form expressions of their ROC curves.

Fig. 4 shows the comparison among the different detectors in a Gaussian channel when the received signal is affected by phase changes owing to data bits using the same parameters as in Fig. 3. The result illustrates that the DPDI, GPDIT and the coherent integration techniques suffer a strong degradation 


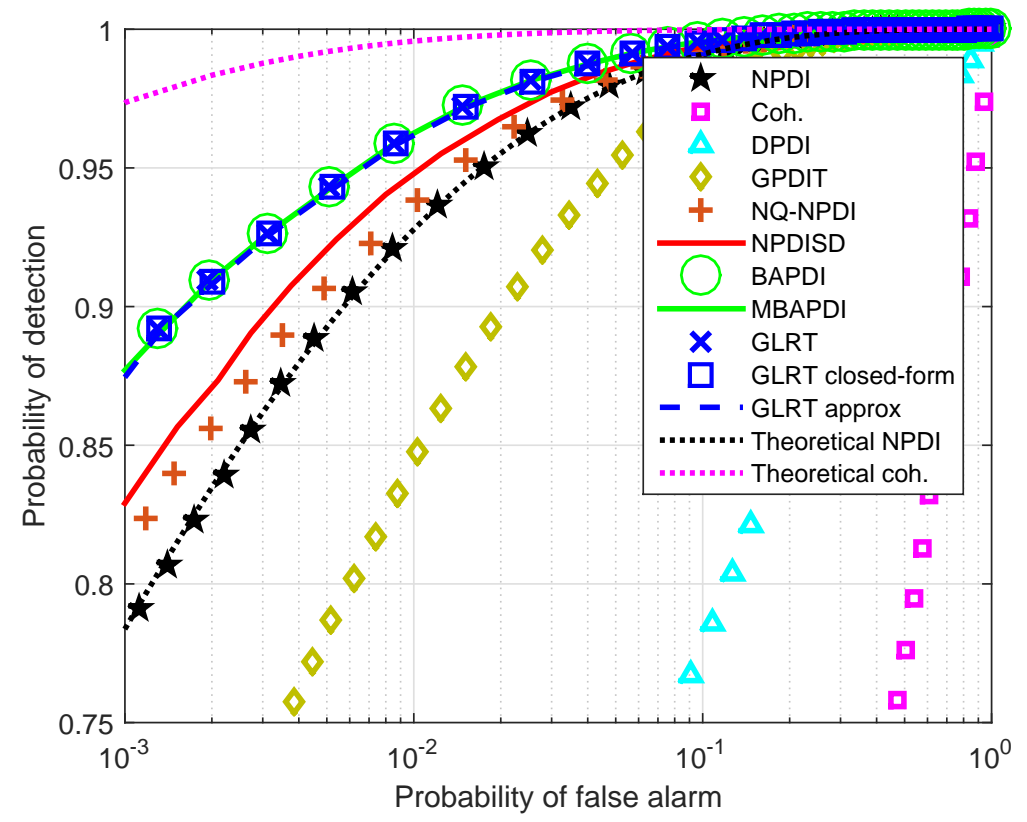

Fig. 4. Performance comparison of the different detectors in presence of data bits for $N_{n c}=6, A=1.6$ and $\sigma=1$. In the legend, GLRT, GLRT closed-form and GLRT approx refer to the techniques explained in Subsections IV-B1 IV-B2 and IV-B3, respectively.

performance since they are not robust against the presence of bits. In this case, the proposed five techniques, two based on the Bayesian approach and three established from the GLRT, provide a very similar performance outperforming the rest of the PDI techniques. In particular, it is interesting to pay attention to the comparison between the proposed five techniques and the NPDISD technique, which was derived by the application of the GLRT approach, but the author used an approximation for a low SNR regime. The outcome reveals a clear improvement in favour of the techniques proposed herein.

Fig. 5 illustrates the comparison among the different detectors in a Gaussian channel when the received signal contains unknown data bits for $A=1$ and $N_{n c}=15$. This simulation reveals that although the SNR of the correlator output is lower than in Fig. 3 and Fig. 4 the proposed five techniques remain exhibiting the best performances. The performance difference among the five techniques and the NPDISD is smaller than in the previous simulations due to this lower SNR value. This value also causes that the technique described in Subsection IV-B3, which has been derived for a relative large values of SNR, has a slight mismatch with respect to the techniques defined in Subsection IV-B1 and Subsection IV-B2, The MBAPDI technique also offers a very slight degradation with respect to the BAPDI because the SNR at the output of the PDI technique is slightly lower than in Fig. 4. This effect can be seen in the zoom 
view, which appears in Fig. 5 .

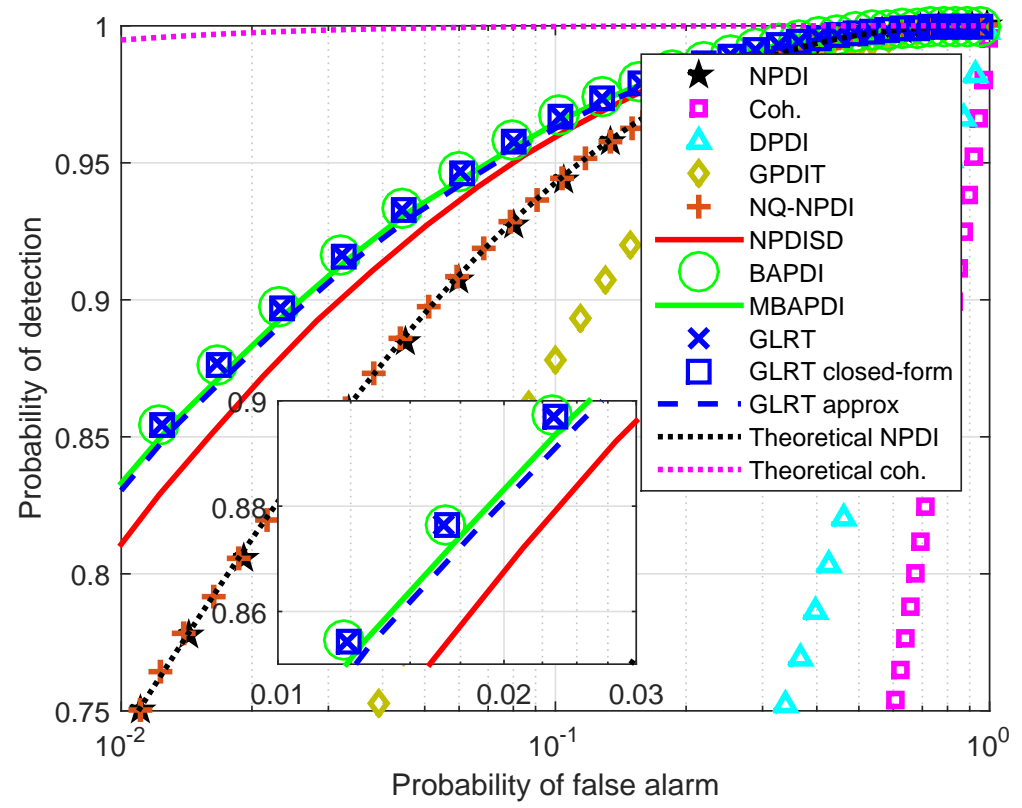

Fig. 5. Performance comparison of the different detectors in presence of data bits for $N_{n c}=15, A=1$ and $\sigma=1$. In the legend, GLRT, GLRT closed-form and GLRT approx refer to the techniques explained in Subsections IV-B1, IV-B2 and IV-B3, respectively.

Fig. 6 shows the probability of detection with respect to the SNR for the different detectors in a Gaussian channel and when the received signal contains data bits. We use $N_{n c}=5$ and set the probability of false alarm to $1 e-3$. The detection threshold for each PDI technique is fixed through the Monte Carlo simulations. The result illustrates that the techniques proposed in the paper show the highest probabilities of detection. The coherent integration, DPDI and GPDIT techniques suffer a severe degradation due to the data bits. For this reason, these techniques are not useful in detection problems where the received signal have sign changes produced by the bits. The NPDI, NQ-NPDI and NPDISD techniques, which are robust against the presence of data bits, outperform the coherent integration, DPDI and GPDIT techniques, but the former group does not provided as good performance as the techniques presented in this work.

Given that the five techniques presented in the paper offer very similar performance, exceeding that of the other techniques, for the problem at hand, the selection of the most suitable one can be based on the computational complexity. While the BAPDI is the theoretically optimal PDI technique since it has been derived from the Bayesian approach, it may present difficulties in practice because it uses a matrix, whose size grows exponentially as $N_{n c}$ grows. Moreover, the BAPDI requires the a priori 


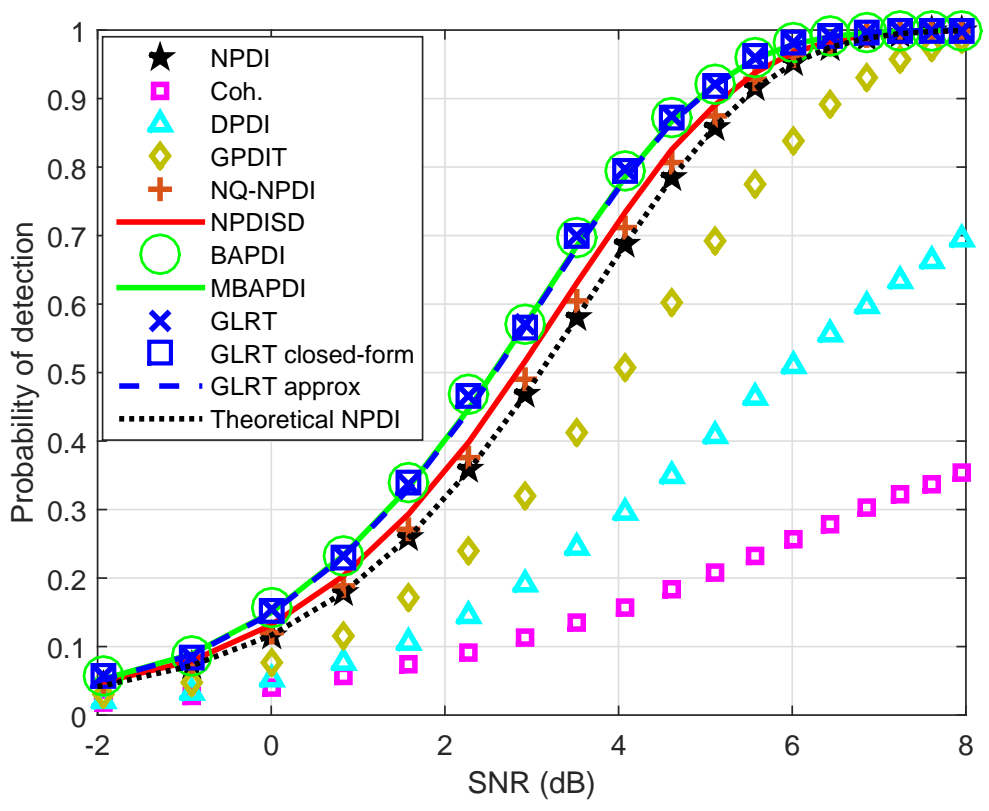

Fig. 6. Probability of detection vs. SNR with $N_{n c}=5$ and probability of false alarm of $1 \mathrm{e}-3$ for the different detectors in presence of data bits in the received signal.

knowledge of the SNR and it needs to use the modified Bessel function, which in practice has to be evaluated numerically. The MBAPDI also suffers the disadvantage of having to evaluate a potentially large number of combinations, which introduces a large computational burden, especially for large $N_{n c}$ values. The exact GLRT presented in Subsection IV-B1 requires the usage of a one-dimensional search method to estimate the phase of the received signal. This fact poses difficulties in the implementation of this technique in a HS-GNSS receiver. The GLRT approach described in Subsection IV-B2 is a good option since it does not depend on large matrices nor a one-dimensional search method, but it has the drawback of requiring the knowledge of the SNR. Finally, the PDI technique presented in Subsection IV-B3 becomes the best option to obtain a significant gain in terms of signal detection because its computational load is the lowest one and it does not need a priori information about the SNR.

\section{CONCLUSIONS}

In this paper we have derived two PDI techniques by using the Bayesian approach and the GLRT for the reacquisition of weak GNSS signals. We have also proposed approximate techniques of reduced computational complexity, which can be easily implemented in HS-GNSS receivers and do not require the knowledge of the SNR. Simulation results have shown the superior performance of the techniques 
proposed in the paper with respect to other PDI techniques, while the former group provides very similar performance. For a balanced trade-off between computational burden and performance, we can conclude that the most suitable technique for the reacquisition of GNSS signals is the one based on the approximation of the GLRT approach for high SNR regime and on the use of the approximate ML phase estimate.

\section{REFERENCES}

[1] G. Seco-Granados, J. Lopez-Salcedo, D. Jimenez-Banos, and G. Lopez-Risueno, "Challenges in indoor global navigation satellite systems: Unveiling its core features in signal processing," IEEE Signal Processing Magazine, vol. 29, no. 2, pp. 108-131, March 2012.

[2] C. Yang, M. Miller, E. Blasch, and T. Nguyen, "Comparative study of coherent, non-coherent, and semi-coherent integration schemes for GNSS receivers," in ION Annual meeting, April 2007, pp. 572-588.

[3] F. Dovis and T. H. Ta, "High sensitivity techniques for GNSS signal acquisition," in Global Navigation Satellite Systems: Signal, Theory and Applications, S. Jin, Ed. InTech, 2012.

[4] A. Schmid and A. Neubauer, "Differential correlation for Galileo/GPS receivers," in IEEE International Conference on Acoustics, Speech, and Signal Processing (ICASSP), vol. 3, March 2005, pp. iii/953-iii/956.

[5] H. Elders-Boll and U. Dettmar, "Efficient differentially coherent code/Doppler acquisition of weak GPS signals," in IEEE Eighth International Symposium on Spread Spectrum Techniques and Applications, Aug 2004, pp. 731-735.

[6] G. Corazza and R. Pedone, "Generalized and average likelihood ratio testing for post detection integration," IEEE Transactions on Communications, vol. 55, no. 11, pp. 2159-2171, Nov 2007.

[7] D. Gómez-Casco, J. A. López-Salcedo, and G. Seco-Granados, "Optimal fractional non-coherent detector for highsensitivity GNSS receivers robust against residual frequency offset and unknown bits," in 14th Workshop on Positioning, Navigation and Communications (WPNC), Oct 2017, pp. 1-5.

[8] S. Satyanarayana, D. Borio, and G. Lachapelle, "A non-coherent block processing architecture for standalone GNSS weak signal tracking," in ION GNSS, September 2011, pp. 1777-1785.

[9] F. S. T. Van Diggelen, A-GPS: Assisted GPS, GNSS, and SBAS. Artech House, 2009.

[10] E. Kaplan and C. Hegarty, Understanding GPS: principles and applications. Artech house, 2005.

[11] D. Gómez-Casco, J. A. López-Salcedo, and G. Seco-Granados, “Generalized Integration Techniques for High-Sensitivity GNSS Receivers Affected by Oscillator Phase Noise," in IEEE Statistical Signal Processing Workshop (SSP), 2016.

[12] D. Borio and G. Lachapelle, "A non-coherent architecture for GNSS digital tracking loops," Annals of Telecommunicationsannales des télécommunications, vol. 64, no. 9-10, pp. 601-614, 2009.

[13] G. Corazza and R. Pedone, "Maximum likelihood post detection integration methods for spread spectrum systems," in IEEE Wireless Communications and Networking (WCNC), 2003, vol. 1, March 2003, pp. 227-232 vol.1.

[14] M. A. Richards, Fundamentals of radar signal processing. Tata McGraw-Hill Education, 2005.

[15] M. Simon, M. Shihabi, and T. Moon, "Optimum detection of tones transmitted by a spacecraft," TDA PR 42-123, pp. 69-98, 1995.

[16] R. N. McDonough and A. D. Whalen, Detection of signals in noise. Academic Press, 1995.

[17] B. C. Levy, Principles of signal detection and parameter estimation. Springer Science \& Business Media, 2008.

[18] D. Rife and R. Boorstyn, "Single tone parameter estimation from discrete-time observations," IEEE Transactions on Information Theory, vol. 20, no. 5, pp. 591-598, Sep 1974. 
[19] C. Jayaram and C. R. Murthy, "Noncoherent integration for signal detection: Analysis under model uncertainties," IEEE Transactions on Aerospace and Electronic Systems, vol. 49, no. 4, pp. 2413-2430, 2013. 